\title{
Developmental changes in the ability to synthesize juvenile hormone in vitro by corpora allata from the Eri silkworm, Samia cynthia ricini (Lepidoptera: Saturniidae)
}

\author{
SHENG LI*, Rong-Jing JIANG and MEI-XuN CAO ${ }^{1}$
}

\begin{abstract}
${ }^{1}$ Shanghai Institute of Entomology, Shanghai Institutes for Biological Sciences, Chinese Academy of Sciences, 225, Chongqing Road (S), Shanghai 200025, China; e-mail: mxcao@public.sta.net.cn
\end{abstract}

Key words. Samia cynthia ricini, developmental changes; corpora allata; juvenile hormone; radiochemical assay

\begin{abstract}
A radiochemical assay (RCA) has been used for the measurement of juvenile hormone (JH) synthesis in vitro by corpora allata (CA) from the Eri silkworm, Samia cynthia ricini. Using CA from newly emerged female adults for the bioassay, the most suitable incubation conditions were determined. A high rate of JH synthesis was found in medium TC199 at $\mathrm{pH} 6.5,30^{\circ} \mathrm{C}$ and $4 \mathrm{mM}$ $\mathrm{Ca}^{2+}$. The time course of $\mathrm{JH}$ synthesis showed a steady decrease during the first 6 hours of incubation.

Under optimal incubation conditions, CA from the 4th and 5th larval instars, pupae and adults were used for measuring JH synthesis in vitro. The highest rates of JH synthesis were found on the 1st day of both larval stages, and then JH synthesis decreased steadily during the following two days of each instar. From the 4th day of the 5th larval instar to the 2nd day after pupation, CA could still synthesize small amounts of JH. However, from the 3rd day of the pupal stage to 12 hours before adult emergence, no $\mathrm{JH}$ release was observed. About 6 hours before emergence, CA of both female and male pharate adults regained the ability to synthesize JH. JH synthesis increased to a maximum shortly after emergence and then decreased again during the following two days. During this period, JH synthesis in vitro by CA from females was always higher than that of males. This is the first report on JH synthesis in vitro by CA from both female and male pharate adults and adults of a lepidopteran species, where the adults do not feed, are relatively short-lived, mate only once, and ovarian maturation and vitellogenesis are completed before emergence.
\end{abstract}

\section{INTRODUCTION}

Juvenile hormone, a unique insect sesquiterpenoid hormone, is produced by the corpora allata (CA). During insect development, juvenile hormone $(\mathrm{JH})$ maintains the juvenile character. Metamorphosis to the pupa or adult form requires a reduced level or absence of $\mathrm{JH}$ (reviewed by Riddiford, 1994). In the adult stage, JH regulates many reproductive functions, including vitellogenesis, spermatogenesis and growth of the accessory sex gland (ASG) (reviewed by Wyatt \& Davey, 1996). The rate of JH synthesis appears to be regulated by stimulating and inhibiting neuropeptides, allatotropins (AT) and allatostatins (AST) (reviewed by Stay et al., 1994; Guan, 1996). Before studying AT and AST in an insect species, it is necessary to optimize the radiochemical assay (RCA) for the measurement of $\mathrm{JH}$ synthesis in vitro by corpora allata (CA) (reviewed by Feyereisen, 1985).

An RCA for JH synthesis was established in 1974 (Pratt \& Tobe, 1974; Tobe \& Pratt, 1974). When assaying glands of a species not previously studied, an optimal culture medium should be identified. Parameters such as $\mathrm{pH}$ and $\mathrm{Na}^{+} / \mathrm{K}^{+}$ratio are very important (reviewed by Feyereisen, 1985; Yagi \& Tobe, 2001). In addition, $\mathrm{Ca}^{2+}$ can act as a second messenger controlling $\mathrm{JH}$ synthesis. This means that the $\mathrm{Ca}^{2+}$ concentration in the medium will also affect JH synthesis (reviewed by Rachinsky \& Tobe, 1996). Using the suggested incubation conditions for CA of the beetle Coccinella septempunctata (Guan \& Chen, 1986), it was found that the rate of JH synthesis in vitro by CA from newly emerged female adult Eri silkworm Samia cynthia ricini was extremely low. In this paper, we present the most suitable incubation conditions for $\mathrm{CA}$ from $S$. cynthia ricini, the time course of $\mathrm{JH}$ synthesis and release, as well as a dose-response curve for methionine (Met).

Eri silkworm S. cynthia ricini, silkworm Bombyx mori, and tobacco hornworm Manduca sexta belong to three closely related families of Lepidoptera: Saturniidae, Bombycidae, and Sphingidae, respectively. The Lepidoptera may be divided into four distinct groups based on their gonadotropic hormones and other reproductive and biological characteristics, regardless of phylogenetic relationships (reviewed by Ramaswamy et al., 1997; Table 1). The silkworms Hyalophora cecropia, S. cynthia ricini and $B$. mori belong to group $\mathrm{I}$, and $M$. sexta belongs to group III. B. mori and $M$. sexta are two important model insect species for studying insect biochemistry and physiology. Developmental changes in their abilities to synthesize $\mathrm{JH}$ in vitro have been studied in detail. We now report on $\mathrm{JH}$ synthesis in vitro by CA from $S$. cynthia ricini 4 th and 5th larval instars, pupae, as well as pharate adults and adults of both sexes. This study is prerequisite for the identification of AT and AS from S. cynthia ricini, which is the main purpose of our research project.

\footnotetext{
* Present address. Department of Biological Science, Illinois State University, Normal, IL, 61790, USA; tel: $+1+309-4383085$; fax: $+1+309+4383722$; e-mail: sli@ilstu.edu
} 


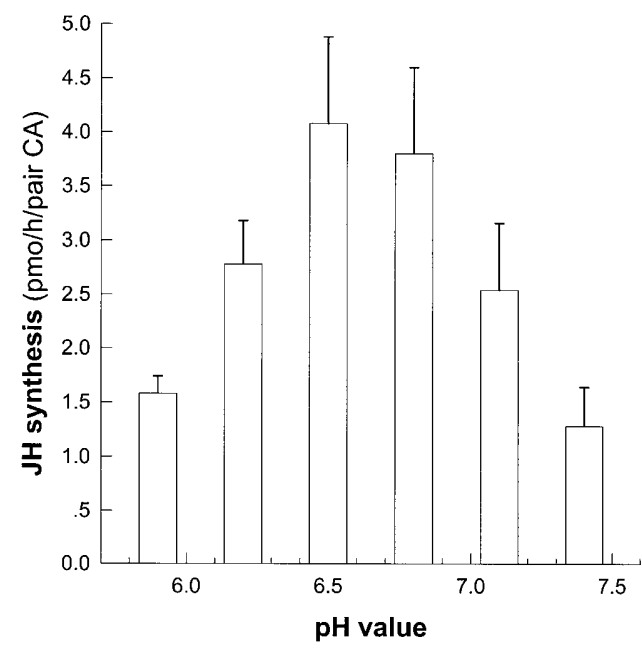

Fig. 1. JH synthesis in vitro by CA from newly emerged female adults of $S$. cynthia ricini at different $\mathrm{pH}$ values. Each point and bar represents the mean $\pm \mathrm{SE}, \mathrm{n}=6$. Compared with JH synthesis at $\mathrm{pH} 6.5, \mathrm{P}>0.05$ at $\mathrm{pH} 6.8, \mathrm{P}<0.05$ at $\mathrm{pH} 6.2$ and $\mathrm{pH} 7.1$, and $\mathrm{P}<0.01$ at $\mathrm{pH} 5.9$ and $\mathrm{pH} 7.4$ (t-test).

\section{MATERIALS AND METHODS}

\section{Animals}

S. cynthia ricini larvae and pupae were received from Zhengjiang, China. Larvae were reared on fresh castor-oil plant leaves at $25 \pm 1{ }^{\circ} \mathrm{C}$ under a $14 \mathrm{~h}$ light $(6: 00-20: 00): 10 \mathrm{~h}$ dark photoperiod. Developmental stages were adjusted at the time of each ecdysis and at wandering in the 5th larval instar. After pupation, the pupae were sexed, and males and females were kept separately under the above rearing conditions until emergence Pharate adults were collected at 24, 12 and 6 hours before emergence for the CA assay. Newly emerged adults (less than 4 hours after emergence), as well as the 1- and 2-day-old adults ( 24 and 48 hours after emergence) were also used.

\section{JH synthesis in vitro by $\mathrm{CA}$}

The suggested incubation conditions for CA of the beetle $C$. septempunctata (Guan \& Chen, 1986) were at first used for incubation of CA from newly emerged female adult $S$. cynthia ricini. Corpora cardiaca-corpora allata complexes (CC-CA) were dissected under a saline solution $(0.111 \mathrm{~g} / 1 \mathrm{NaCl}, 3.355 \mathrm{~g} / \mathrm{l}$ $\mathrm{KCl}, 0.555 \mathrm{~g} / \mathrm{l} \mathrm{CaCl}$, and $5.083 \mathrm{~g} / 1 \mathrm{MgCl}_{2}, \mathrm{pH} 6.5$ ), and placed into medium TC199 (Gibco). The interval between extirpation of CC-CA and the commencement of incubation in the presence of radiolabeled methionine (Met) was about $30 \mathrm{~min}$. One pair of CC-CA were incubated in $100 \mu \mathrm{l}$ medium TC199 (KangDa, with L-Glu, Hank's salts and 25 mM HEPES buffer, without $\mathrm{CaCl}_{2}$ and Met, $\mathrm{pH}$ 7.2) containing $1 \mu \mathrm{Ci}{ }^{3} \mathrm{H}-\mathrm{Met}$ (NEN; specific activity $100 \mathrm{mCi} / \mathrm{mmol}$; initial concentration $10 \mathrm{mM}$; final concentration $100 \mu \mathrm{M}$ ), $1.3 \mathrm{mM} \mathrm{CaCl}, 0.2 \%$ Ficoll (type 400, Sigma), and $0.1 \%$ bovine serum albumin (Sigma). Routinely, gland pairs were randomized into small groups and incubated in the dark for 3 hours at $30^{\circ} \mathrm{C}$ with gentle agitation. Then, $300 \mu \mathrm{l}$ isooctane was added to terminate the incubation. After vortexing and centrifugation, $200 \mu \mathrm{l}$ supernatant was used for measuring radioactivity (DPM) in an LS6500 scintillation counter (Beckman) using a scintillation cocktail of toluene + $0.7 \%$ PPO $+0.05 \%$ POPOP.

Additionally, some material extracted with isooctane was subjected to NP-HPLC and RP-HPLC separation. Radiolabeled JH I, II and III were found, but JH II was the main JH released by $\mathrm{CA}$ of this insect species ( $\mathrm{Li}$ et al., unpublished data).
In order to obtain the optimal rate of $\mathrm{JH}$ synthesis and release, the incubation conditions in medium TC199 ( $\mathrm{pH}$ value, temperature, $\mathrm{Ca}^{2+}$ concentration, $\mathrm{Na}^{+} / \mathrm{K}^{+}$) were modified as follows: $\mathrm{pH}$ values of $5.9,6.2,6.5,6.8,7.1$, and 7.4 at $4 \mathrm{mM}$ or 1.3 $\mathrm{mM} \mathrm{Ca}{ }^{2+}$ concentration; temperature at $20,23,25,28$, and $30^{\circ} \mathrm{C}$ at $\mathrm{pH} 6.5$ and $4 \mathrm{mM} \mathrm{Ca}^{2+}$ concentration; $\mathrm{Ca}^{2+}$ concentration of 0 , $0.5,1,2,4$, and $8 \mathrm{mM}$ at $\mathrm{pH} 6.5$ and $30^{\circ} \mathrm{C}$. Under the most suitable conditions derived from the above experiments, three experimental groups were tested: 1 , addition of $10 \mu \mathrm{l}$ of $0.9 \%$ $\mathrm{NaCl} ; 2$, incubation for 3 hours in light; 3 , incubation of single CA instead of the CC-CA.

Under the most suitable incubation conditions, the time course of JH synthesis was followed over 6 hours of incubation with different glands for each period, and a dose-response curve for Met was established at concentrations of 2.5, 5, 10, 20, 50, 100 , and $200 \mu \mathrm{M}$.

\section{Bioassay for developmental changes}

CC-CA were dissected from both 4th and 5th instar larvae and from pupae in daily intervals at 9:00. CC-CA were also isolated from the 4th instar larvae and during the first 3 days of the 5th larval stages at PM 21:00. All pupae and adults were sexed. CC-CA were dissected from pharate adults at 24, 12 and 6 hours before emergence, from newly emerged adults, as well as from 1- and 2-day-old adults of both sexes.

\section{RESULTS}

\section{Improvement of incubation conditions in medium TC199}

\section{Effect of pH on JH synthesis}

In all of the following experiments except those of "Developmental changes in JH synthesis", the assayed glands were from newly emerged female adults.

The influence of extra-glandular $\mathrm{pH}$ on the rate of $\mathrm{JH}$ synthesis was investigated by modifying the $\mathrm{pH}$ of medium TC199 over the range of 5.9-7.4 at $4 \mathrm{mM} \mathrm{Ca}^{2+}$. JH synthesis showed an optimum at $\mathrm{pH} 6.5$, which corresponds to the $\mathrm{pH}$ of $S$. cynthia ricini hemolymph. Below or above $\mathrm{pH} 6.5$, the rates of $\mathrm{JH}$ synthesis were drastically reduced (Fig. 1). At all $\mathrm{pH}$ values tested, rates of JH synthesis at $1.3 \mathrm{mM} \mathrm{Ca}^{2+}$ were less than half of that at $4 \mathrm{mM}$ $\mathrm{Ca}^{2+}$ (data not shown). A pH of 6.5 was used in subsequent assays.

\section{Effect of temperature on JH synthesis}

Temperature has a great influence on the rate of $\mathrm{JH}$ synthesis over the range of $20-30^{\circ} \mathrm{C}$. At $20^{\circ} \mathrm{C}$, the rate of $\mathrm{JH}$ synthesis was as high as at $30^{\circ} \mathrm{C}$, whereas $\mathrm{JH}$ synthesis was much lower at $23-25^{\circ} \mathrm{C}$ (Fig. 2). Incubation at $30^{\circ} \mathrm{C}$ was used in subsequent assays.

\section{Effect of $\mathrm{Ca}^{2+}$ concentration on $\mathrm{JH}$ synthesis}

The $\mathrm{Ca}^{2+}$ concentration in Medium TC199 affected the rate of $\mathrm{JH}$ synthesis (Fig. 3). Without $\mathrm{Ca}^{2+}$ in the medium, the rate of $\mathrm{JH}$ synthesis was very low. At least some of the CC-CA did not synthesize $\mathrm{JH}$ at all. In general, higher $\mathrm{Ca}^{2+}$ concentrations resulted in higher rates of $\mathrm{JH}$ synthesis over the range of $0-8 \mathrm{mM} \mathrm{Ca}^{2+}$. Between 0 and 1 $\mathrm{mM} \mathrm{Ca}{ }^{2+}$, the rate increase was linear. At 4-8 $\mathrm{mM} \mathrm{Ca}^{2+}$ in the medium, the rate of $\mathrm{JH}$ synthesis was about 10 times that without $\mathrm{Ca}^{2+}$. Four $\mathrm{mM} \mathrm{Ca}{ }^{2+}$ was used in subsequent experiments. 


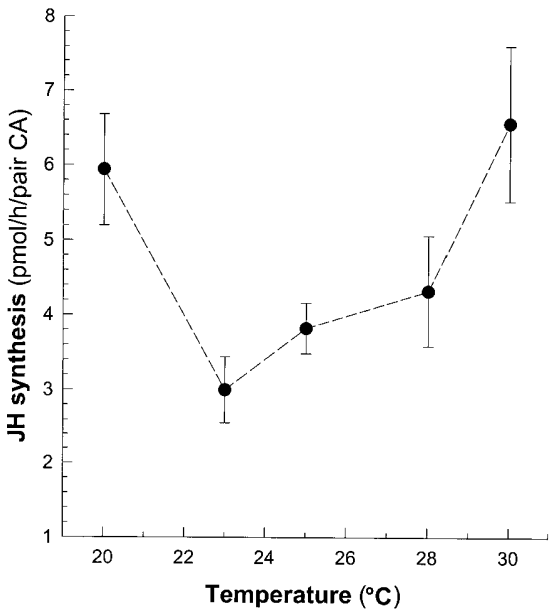

Fig. 2. JH synthesis in vitro by $\mathrm{CA}$ from newly emerged female adults of $S$. cynthia ricini at different temperatures. Each point and bar represents the mean $\pm \mathrm{SE}, \mathrm{n}=6$. Compared with $\mathrm{JH}$ synthesis at $30^{\circ} \mathrm{C}, \mathrm{P}>0.05$ at $20^{\circ} \mathrm{C}, \mathrm{P}<0.01$ at 25 and $28^{\circ} \mathrm{C}$, and $\mathrm{P}<0.001$ at $23^{\circ} \mathrm{C}$ (t-test).

\section{Additional factors affecting JH synthesis}

When $10 \mu 1$ of $0.9 \% \mathrm{NaCl}$ was added to the incubation medium to raise the $\mathrm{Na}^{+} / \mathrm{K}^{+}$ratio, no difference in the rate of JH synthesis between the experimental and the control group was found. Single CA carefully dissected from $\mathrm{CC}-\mathrm{CA}$ showed similar rates of $\mathrm{JH}$ synthesis as the intact complex. In subsequent experiments, $\mathrm{CC}$-CA were incubated. Incubation of CC-CA in light resulted in a rate of $\mathrm{JH}$ synthesis which was only half of that in the dark (data not shown).

\section{Time course of $\mathbf{J H}$ synthesis}

Under the most suitable incubation conditions, a time course of $\mathrm{JH}$ synthesis was determined for the first 6 hours of incubation. Fig. 4 represents JH synthesis on a cumulative basis. We found that $\mathrm{JH}$ synthesis decreased during the six hours of incubation. The rate of JH synthesis was highest during the 1 st hour, but nearly no $\mathrm{JH}$ was released after 4 hours of incubation. CA were incubated for 3 hours in subsequent experiments.

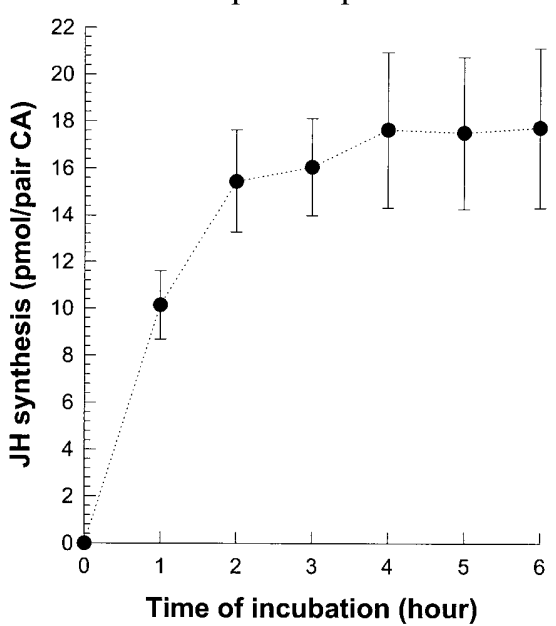

Fig. 4. JH synthesis in vitro by CA from newly emerged female adults of $S$. cynthia ricini during 6 hours of incubation. Each point and bar represents the mean $\pm \mathrm{SE}, \mathrm{n}=6$.

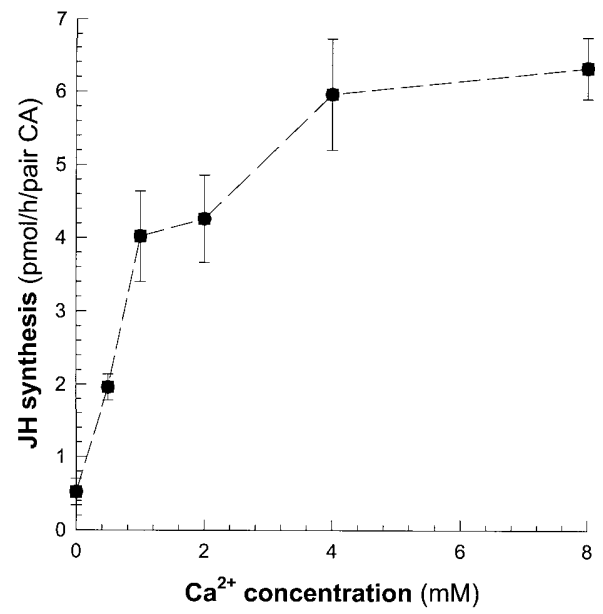

Fig. 3. JH synthesis in vitro by $\mathrm{CA}$ from newly emerged female adults of $S$. cynthia ricini at different $\mathrm{Ca}^{2+}$ concentrations. Each point and bar represents the mean $\pm \mathrm{SE}, \mathrm{n}$ $=6$. Compared with $\mathrm{JH}$ synthesis at $8 \mathrm{mM} \mathrm{Ca}^{2+}, \mathrm{P}>0.05$ at 4 $\mathrm{mM} \mathrm{Ca}{ }^{2+}, \mathrm{P}<0.01$ at $1 \mathrm{mM}$ and $2 \mathrm{mM} \mathrm{Ca}^{2+}$, and $\mathrm{P}<0.001$ at 0 and $0.5 \mathrm{mM} \mathrm{Ca}^{2+}$ (t-test).

\section{Dose-response curve for Met}

The influence of Met on the rate of JH synthesis was investigated by incubating glands in medium TC199 containing various concentrations of ${ }^{3} \mathrm{H}$-Met over the range of 2.5 to $200 \mu \mathrm{M}$. Fig. 5 shows that the highest rates of $\mathrm{JH}$ synthesis were obtained at Met concentrations ranging from 50 to $200 \mu \mathrm{M}$. Between 2.5 and $20 \mu \mathrm{M}$ Met, the rates of $\mathrm{JH}$ synthesis increased linearly. $50 \mu \mathrm{M}$ Met was used in subsequent experiments.

\section{Developmental changes in $\mathrm{JH}$ synthesis \\ Changes in JH synthesis in the 4th larval instar}

The 4th larval instar lasts for 3 days. CC-CA were extirpated and incubated at AM 9:00. JH synthesis in vitro by CA from newly ecdysed (the 1st day) larva of this instar was high $(0.74 \mathrm{pmol} / \mathrm{h} / \mathrm{pair} \mathrm{CA})$, then decreased steadily over the 2 nd $(0.48 \mathrm{pmol} / \mathrm{h} /$ pair CA $)$ and $3 \mathrm{rd}(0.28 \mathrm{pmol} / \mathrm{h} /$ pair CA) days (Fig. 6).

$\mathrm{JH}$ synthesis was lower at 21:00 than at 9:00 AM of the 1 st and 2 nd day, respectively (data not shown). Therefore, from the 1 st day to the 3 rd day, JH synthesis decreased continuously, and did not show a change correlated with the time of day. However, at 21:00 PM of the 3rd day, when the 4th larval instar was preparing for ecdysis, JH synthesis was much higher $(0.64 \mathrm{pmol} / \mathrm{h} /$ pair CA) than at 9:00 AM of the same day.

\section{Changes in $\mathbf{J H}$ synthesis in the 5th larval instar}

There are 11 days in the 5th larval instar, and wandering starts at day 7 . Newly ecdysed (the 1st day) 5th larval instar showed the highest $\mathrm{JH}$ synthesis in vitro (1.18 $\mathrm{pmol} / \mathrm{h} /$ pair CA), and JH synthesis decreased dramatically during the next 3 days (Fig. 6). From the 4th day to the 7th day (wandering), $\mathrm{JH}$ synthesis decreased only slightly and reached the lowest value at day 7. A drop during the last two days of the 5th larval stadium was followed by a small increase in $\mathrm{JH}$ synthesis at day 9 . 


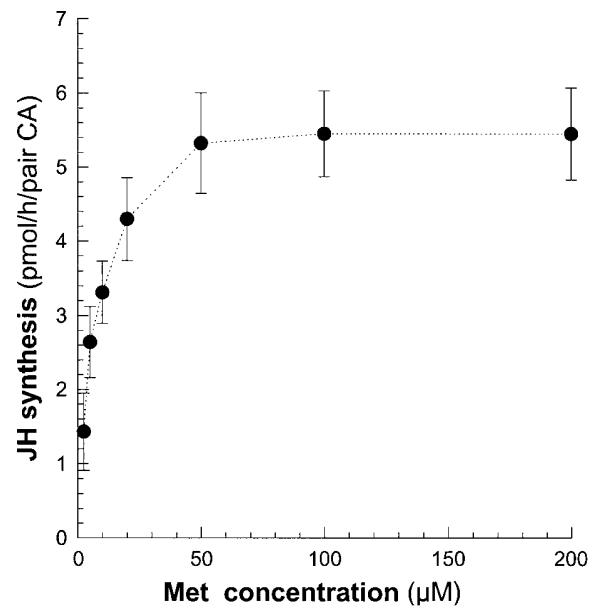

Fig. 5. JH synthesis in vitro by $\mathrm{CA}$ from newly emerged female adults of $S$. cynthia ricini at different Met concentrations. Each point and bar represents the mean $\pm \mathrm{SE}$, $\mathrm{n}=6$. Compared with $\mathrm{JH}$ synthesis at $50 \mu \mathrm{M}$ Met, $\mathrm{P}>0.05$ at $100 \mu \mathrm{M}$ and $200 \mu \mathrm{M}$ Met, $\mathrm{P}<0.05$ at $20 \mu \mathrm{M}$ Met, $\mathrm{P}<0.01$ at $10 \mu \mathrm{M}$ Met, and $\mathrm{P}<0.001$ at $2.5 \mu \mathrm{M}$ Met (t-test).

From the 4 th to the 11 th day of this instar, JH synthesis varied between 0.11 and $0.20 \mathrm{pmol} / \mathrm{h} /$ pair CA.

As in the 4th larval instar, JH synthesis in vitro by CA did not show a change correlated with the time of day during the first 4 days of the 5th larval instar. JH synthesis and release at 21:00 was always lower than at 9:00 of the same day.

\section{Changes in JH synthesis in pupa}

There are about 16 days in the pupal stage. CA still synthesized a very small amount of $\mathrm{JH}(0.07 \mathrm{pmol} / \mathrm{h} /$ pair CA) during the first 2 days of the pupal stage (Fig. 6), but no $\mathrm{JH}$ synthesis was observed from day 3 to day $6 / 7$ of this stadium. It is very difficult to dissect intact CC-CA between day 7 and day 12 of the pupal stage. CA did not synthesize $\mathrm{JH}$ from day 12 until 12 hours before emergence. Six hours before emergence, $\mathrm{CA}$ from both female and male adults regained their ability to synthesize $\mathrm{JH}$ (Fig. 7).

\section{Changes in JH synthesis in pharate adult and adult females and males}

Pharate adults and adults were sexed and kept separately to prevent copulation. At 24 hours before emergence, a few black spots appear at the stethidium of the pupa (pharate adult), but the body is still hard. At 12 hours before emergence, many more black patterns form and the body begins to soften. CA of both female and male pharate adults at 24 to 12 hours before emergence did not synthesize JH. At 6 hours before emergence, all the stethidium of the pharate adult has blackened, some brown patterns also appear at the abdomen, and the body has become completely soft. At this time, CA from both female and male pharate adults regained their ability to synthesize JH. Female pharate adults synthesized 4.82 $\mathrm{pmol} / \mathrm{h} /$ pair CA, males only $0.10 \mathrm{pmol} / \mathrm{h} /$ pair CA. Once $\mathrm{CA}$ regained the ability to synthesize $\mathrm{JH}, \mathrm{JH}$ synthesis increased and reached a maximum at the time of emer-

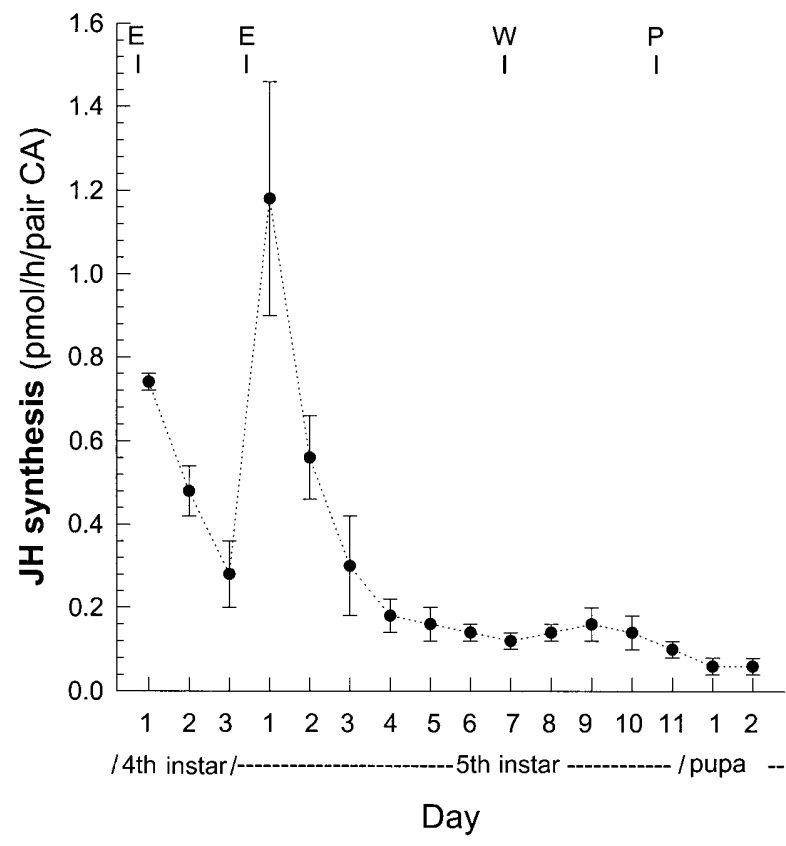

Fig. 6. JH synthesis in vitro by $\mathrm{CA}$ from $4^{\text {th }}$ and $5^{\text {th }}$ instar larvae and early pupae. Each point and bar represents the mean $\pm \mathrm{SE}, \mathrm{n}=6$. Compared with JH synthesis at the $9^{\text {th }}$ day of the $5^{\text {th }}$ instar ranging from the $4^{\text {th }}$ day of the $5^{\text {th }}$ instar to the $2^{\text {nd }}$ day of the pupa stage, $\mathrm{P}>0.05$ at the $4^{\text {th }}, 5^{\text {th }}, 6^{\text {th }}, 8^{\text {th }}$, and $10^{\text {th }}$ day, $\mathrm{P}<$ 0.05 at the $7^{\text {th }}$ and $11^{\text {th }}$ day of the $5^{\text {th }}$ instar, and $\mathrm{P}<0.01$ at the first 2 days of the pupa stage (t-test). E - ecdysis; W - wandering; $\mathrm{P}$ - pupation.

gence ( $0-4$ hours after emergence). JH synthesis of young female adults was $6.56 \mathrm{pmol} / \mathrm{h} /$ pair $\mathrm{CA}$ and males 1.27 $\mathrm{pmol} / \mathrm{h} /$ pair CA. During the following two days, JH synthesis of both females and males decreased steadily and rapidly. At 24 and 48 hours after emergence, JH synthesis was 3.44 and $1.19 \mathrm{pmol} / \mathrm{h} /$ pair CA in females, and 0.32 and $0 \mathrm{pmol} / \mathrm{h} /$ pair CA in males, respectively (Fig. 7).

In general, $\mathrm{CA}$ of both female and male pharate adults regained their ability to synthesize $\mathrm{JH}$ at approximately 6 hours before emergence, and $\mathrm{JH}$ synthesis increased to a maximum at the time of emergence, but later decreased. At this time, CA of female pharate adults and adults synthesized much more $\mathrm{JH}$ than did those of male pharate adults and adults.

\section{DISCUSSION}

The development of an RCA for measuring JH synthesis in vitro by $\mathrm{CA}$ has greatly facilitated research in various areas of $\mathrm{JH}$ biochemistry and physiology. In this assay, medium TC199 was first chosen as an optimal medium for the desert locust Schistocerca gregaria (Tobe \& Pratt, 1974), then used for other insect species (Guan \& Chen, 1986, Weaver et al., 1980), including Lepidoptera, M. sexta (Kramer \& Law, 1980) and the corn earworm, Heliothis armigera (Guan et al., 1995). In this study, we used medium TC199 for the RCA in S. cynthia ricini. In general, $\mathrm{pH}$ value, $\mathrm{Na}^{+} / \mathrm{K}^{+}$ratio and $\mathrm{Ca}^{2+}$ concentration are considered to be the most important parameters of the 


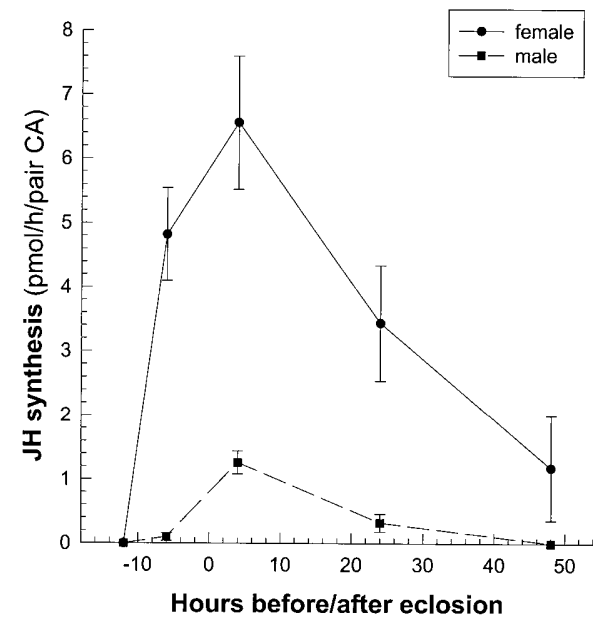

Fig. 7. JH synthesis in vitro by $\mathrm{CA}$ of pharate adults and 1and 2-day-old adults of both sexes. Each point and bar represents the mean $\pm \mathrm{SE}, \mathrm{n}=6$. In both females and males, compared with $\mathrm{JH}$ synthesis at the time of emergence, $\mathrm{P}<0.01$ at all other times. Between females and males, $\mathrm{P}<0.01$ at all stages except 12 hours before emergence (t-test).

medium (reviewed by Feyereisen, 1985; Yagi \& Tobe, 2001). The $\mathrm{pH}$ values of 7.2 and 6.5 , the same $\mathrm{pH}$ as in the hemolymph, were used in Tenebrio molitor (Weaver et al., 1980) and M. sexta (Kramer \& Law, 1980), respectively. It is not surprising that the most suitable $\mathrm{pH}$ in $S$. cynthia ricini was 6.5 , also the same $\mathrm{pH}$ as found in the hemolymph. However, it appears that the $\mathrm{Na}^{+} / \mathrm{K}^{+}$ratio does not have a great influence on $\mathrm{JH}$ synthesis in this insect species. It has been reported that $\mathrm{Ca}^{2+}$ can function as a second messenger in regulating $\mathrm{JH}$ synthesis (reviewed by Rachinsky \& Tobe, 1996). When CA from $S$. gregaria (Thompson \& Tobe, 1986), M. sexta (Allen et al., 1992), and cockroach Diploptera punctata (Kikukawa et al., 1987) were incubated in the absence of $\mathrm{Ca}^{2+}$, little or no JH was synthesized. In D. punctata, JH synthesis correlated with $\mathrm{Ca}^{2+}$ concentration in a linear manner and was highest at 3-5 $\mathrm{mM} \mathrm{Ca}^{2+}$ (Kikukawa et al., 1987). A similar dose-response curve of $\mathrm{Ca}^{2+}$ concentration was found in this study for S. cynthia ricini. Few data are available on the influence of temperature on $\mathrm{JH}$ synthesis. In most studies, $25^{\circ} \mathrm{C}$ or $30^{\circ} \mathrm{C}$ was chosen for the bioassay. However, in S. cynthia ricini, the rate of $\mathrm{JH}$ synthesis varied greatly over the temperature range of $20-30^{\circ} \mathrm{C}$. Both pupae and adults were kept at $25^{\circ} \mathrm{C}$, but the rates of $\mathrm{JH}$ synthesis were lowest at this temperature. It is difficult to understand why JH synthesis was lowest at the rearing temperature of the animals. In most insect species, including M. sexta (Kramer \& Law, 1980) and C. septempunctata (Guan \& Chen, 1986), JH synthesis was rather constant for several hours of incubation. However, a quite different time course of $\mathrm{JH}$ synthesis was seen in $S$. cynthia ricini. CA from this species synthesized $\mathrm{JH}$ in vitro for only about 3-4 hours. A similar time course was observed in T. molitor (Weaver et al., 1980), although the decrease in $\mathrm{JH}$ synthesis was not so rapid. It is possible that there are not enough precursors in the CA. In summary, high rates of $\mathrm{JH}$ synthesis were found at $\mathrm{pH} 6.5$, $30^{\circ} \mathrm{C}$, a $\mathrm{Ca}^{2+}$ concentration of $4 \mathrm{mM}$, and a methionine concentration of $50 \mu \mathrm{M}$.

S. cynthia ricini, B. mori and $M$. sexta belong to three closely related families of Lepidoptera (Table 1). The developmental changes in $\mathrm{JH}$ synthesis of these three insect species are compared below. Both similarities and differences have been found. No great differences in the developmental changes of $\mathrm{JH}$ hemolymph titers were observed between $M$. sexta and $B$. mori larvae. In $M$. sexta, high $\mathrm{JH}$ hemolymph titers were found at the beginning of the 4th (Hidayat \& Goodman, 1994) and 5th larval instar (Baker et al., 1987). Another JH peak appeared at day 6 or 7 of the last larval stadium, 1 or 2 days after wandering (Baker et al., 1987). Similar developmental changes of $\mathrm{JH}$ hemolymph titers were seen in $B$. mori. In B. mori, however, JH hemolymph titers changed with photoperiod. At the end of each larval instar, JH titer started to increase concomitantly with ecdysis, which occurred during the photophase, and the peak titer was found at the end of the same photophase (Niimi \& Sakurai, 1997).

In $M$. sexta 5th larval instar, developmental changes in $\mathrm{JH}$ synthesis in vitro did not completely correspond to the changes in $\mathrm{JH}$ hemolymph titers. High rates of JH synthesis in vitro were observed during the first two days of the 5th larva instar. However, the CA did not synthesize any $\mathrm{JH}$ at the 4th and 5th day of this instar (Kramer \& Law, 1980). The reason why CA cannot synthesize JH at this time is the absence of methyltransferase activity, thus $\mathrm{JH}$ acid cannot be converted to $\mathrm{JH}$. It was discussed that $\mathrm{JH}$ and $\mathrm{JH}$ acid (in males) were synthesized by imaginal discs during the period of wandering in this insect species (Sparagana et al., 1984; 1985). In B. mori, rates of JH synthesis by CA in vitro were similar to those in $M$. sexta (Niimi \& Sakurai, 1997). However, it is still unclear whether imaginal discs in B. mori can synthesize $\mathrm{JH}$ and $\mathrm{JH}$ acid.

TABLE 1. Aspects of reproductive biology of four groups of Lepidoptera.

\begin{tabular}{lccccc}
\hline Type & Species & Egg development stage & Gonadotropic hormone & Adult feeding & Mating times \\
\hline I & Bombyx mori & Larva & Ecd & No & Single \\
& Hyalophora cecropia & Larva/pupa & None & No & Single \\
& Samia cynthia ricini & Larva/pupa & None & No & Single \\
II & Plodia interpunctella & Pupa & Declining Ecd & No & Single \\
III & Manduca sexta & Pupa/adult & JH (chorionation only) & Yes & Several \\
IV & Helicoverpa zea & Adult & JH & Yes & Several \\
& Heliothis virescens & Adult & JH & Yes & Several \\
\hline
\end{tabular}

After Ramaswamy et al., 1997. Ecd = ecdysteroids. 
In summary, a peak of $\mathrm{JH}$ synthesis or $\mathrm{JH}$ hemolymph titer occurs at the beginning of each larval instar in all three insect species. In $B$. mori, there are circadian changes in $\mathrm{JH}$ synthesis and $\mathrm{JH}$ hemolymph titer, but no changes in JH to photoperiod were observed in S. cynthia ricini and $M$. sexta. Similar developmental changes in $\mathrm{JH}$ synthesis were found in the 5th larval instar of both $S$. cynthia ricini and $B$. mori. Besides the peak at the beginning of this instar, there is a small peak of $\mathrm{JH}$ synthesis just after wandering. However, in S. cynthia ricini this peak is much smaller than in B. mori, and is not significant. In $M$. sexta, CA cannot synthesize JH from day 4 of this stadium to the day of pupation. From these results, it seemed worthwhile to investigate the developmental changes in JH hemolymph titers of $S$. cynthia ricini. We have developed both NP-HPLC and RP-HPLC to detect the nature of $\mathrm{JH}$ released by $\mathrm{CA}$ in vitro, and found that JH I, II and III were released into the medium and JH II was the main JH (unpublished data). Similar results were obtained in parasitized and non-parasitized Heliothis virescens ( $\mathrm{Li}$ and Pennacchio, in press), where the same HPLC separation methods were applied.

CA from $M$. sexta female adults synthesized JH in high amounts, but CA from male adults did not (Kramer \& Law, 1980). Further studies showed that there was a reactivation mechanism of $\mathrm{CA}$ in pharate adult $M$. sexta. About 18 hours before emergence, CA from female pharate adults were reactivated and began to synthesize $\mathrm{JH}$ until several days into the adult stage. CA from male pharate adults at the same time did not synthesize $\mathrm{JH}$, but rather $\mathrm{JH}$ acid. This situation was maintained until several days after emergence. The reason why CA cannot synthesize $\mathrm{JH}$ in male pharate adults and adults is that again no methyltransferase activity is present, which converts $\mathrm{JH}$ acid to JH (Hebda et al., 1994).

In general, CA cannot synthesize $\mathrm{JH}$ in both female and male pharate adults and adults of the Lepidopteran group I, including $B$. mori and $H$. cecropia (reviewed by Ramaswamy et al., 1997). CA from adult Saturniidae $H$. cecropia did not synthesize JH (reviewed by Ramaswamy et al., 1997), although a great amount of JH-like substance was extracted from its abdomen (Kramer \& Law, 1980). How can CA from pharate adults and adults of $S$. cynthia ricini synthesize JH? It is possible that a similar reactivation mechanism of CA exists in S. cynthia ricini pharate adults as in $M$. sexta. Nevertheless, several differences in JH synthesis in vitro were seen between these two species. The greatest difference was that CA from male pharate adults and adults of $S$. cynthia ricini synthesized small amounts of $\mathrm{JH}$, but CA from $M$. sexta did not. Another difference was that the ability to synthesize $\mathrm{JH}$ was maintained for a much shorter time, and changes were more rapid in $S$. cynthia ricini females than in $M$. sexta. In fact, it is very difficult to distinguish CA between 12 and 6 hours before emergence by their outward appearances, each gland is large and transparent. In S. cynthia ricini, Mas-AT and AT factor cannot reactivate non-activated $\mathrm{CA}$ at 24 hours before emergence to synthesize $\mathbf{J H}$ at all, but can partially induce CA at 12 hours before emergence to synthesize a small amount of $\mathrm{JH}$ (0.1-0.4 pmol/h/pair CA, unpublished data). So it seems that Mas-AT and AT factor are involved in CA reactivation in this insect speices. However, in M. sexta, Mas-AT did not induce $\mathrm{JH}$ production in inactive female $\mathrm{CA}$ and did not increase methyltransferase activity at all, and it was concluded that reactivation of CA was a process distinct from stimulation of $\mathrm{JH}$ production (Hebda et al., 1994). It is very interesting that such a great difference exists between these two insect species. But why does $\mathrm{JH}$ synthesis by CA change so quickly? There is no doubt that some stimulating and inhibiting neuropeptides exist which regulate JH synthesis in $S$. cynthia ricini. AT and AS factors have been found in brain extracts during the process of purification ( $\mathrm{Li}$ et al., in press).

In the early 1960s, a great amount of a JH-like substance was extracted from the abdomens of male adult $S$. cynthia ricini in our laboratory. Injection of a JH-like substance to early pupa (not longer than 48 hours after pupation) resulted in a second pupation and pupa-adult metamorphosis occurred later (Cao et al., 1963). We are now investigating if accessory genital gland (ASG) in male $S$. cynthia ricini can synthesize $\mathrm{JH}$, or it can only convert $\mathrm{JH}$ acid to $\mathrm{JH}$, and the main location of the source of JH in the abdomen of male adult $S$. cynthia ricini.

In general, it is believed that CA isolated from both female and male pharate adults and adults of Lepidoptera group I animals (where the adults do not feed and are relatively short-lived, mate only once, and ovarian maturation and vitellogenesis are completed before emergence) cannot synthesize JH (reviewed by Ramaswamy et al., 1997). The results of our studies on pharate adults and adults of $S$. cynthia ricini contradict the above conclusion. Therefore, how JH functions might be worthy of further studies in both female and male pharate adults and adults of S. cynthia ricini. It is possible that $\mathrm{JH}$ may trigger oviposition in females and stimulate copulation in males. We are now using classical physiological techniques, such as allatectomy, ASGectomy, and $\mathrm{JH}$ or $\mathrm{JH}$ analog treatment to test these hypotheses.

ACKNOWLEDGEMENTS. This work was supported by the National Natural Science Foundation of China, and Shanghai Institute of Biological Sciences, Chinese Academy of Sciences. We thank Prof. Dr. Klaus H. Hoffmann (Bayreuth, Germany) for his helpful suggestions for the improvement of the manuscript. We also thank Dr. Taub-Montenmayor (U. Texas, USA) for suggestions for improvement.

\section{REFERENCES}

Allen C.U., Janzen W.P. \& Granger N.A. 1992: Manipulation of intercellular calcium affects in vitro juvenile hormone synthesis by larval corpora allata of Manduca sexta. Mol. Cell. Endocrin. 84: 227-241.

Baker F.C., Tsai L.W., Reuter C.C. \& Schooley D.A. 1987: In vivo fluctuation of $\mathrm{JH}, \mathrm{JH}$ acid, and $\mathrm{JH}$ esterase activity, during development of fifth instar Manduca sexta. Insect Biochem. 17: 989-996.

Cao M.X., Zhou G.Z. \& Jiang T.J. 1963: Studies on the effects of juvenile hormone on the pupae of polyvoltine Eri silkworm Attacus ricini. Acta Biol. Exp. Sin. 8: 538-545. 
Feyereisen R. 1985: Radiochemical assay for juvenile hormone III biosynthesis in vitro. Meth. Enzym. 111: 530-539.

GuAN X.C. 1996: Recent advances in research on insect neuropeptides AS and AT. Acta Entomol. Sin. 39: 214-218.

Guan X.C. \& Chen E.Y. 1986: Corpus allatum activity in the female Coccinella septempunctata L. adults. Acta Entomol. Sin. 29: 10-15.

Guan X.C., OUYANG Y.C. \& Zru D.H. 1995: Identification and quantification of juvenile hormones biosynthesis in vitro by corpora allata of two Lepidopterans. Sinozoologia 12: 38-43.

Hebda C., Yu J.H., Bhaskaran G. \& Dahm K.H. 1994: Reactivation of corpora allata in pharate adult Manduca sexta. $J$. Insect Physiol. 40: 849-858.

Hidayat P. \& GoOdman W.G. 1994: Juvenile hormone and hemolymph juvenile hormone binding protein titers and their interaction in the hemolymph of fourth instar Manduca sexta. Insect Biochem. Mol. Biol. 24: 709-715.

Kirukawa S., Tobe S.S., Solowiej S., Rankin S.M. \& Stay B. 1987. Calcium as a regulator of juvenile hormone biosynthesis and release in the cockroach Diploptera punctata. Insect Biochem. 17: 179-187.

KRAMER S.J. \& LAW J.H. 1980: Control of juvenile hormone production: the relationship between precursor supply and hormone synthesis in the tobacco hornworm, Manduca sexta. Insect Biochem. 10: 569-575.

NIIMI S. \& SAKURAI S. 1997: Development changes in juvenile hormone and juvenile hormone acid titers in the hemolymph and in vitro juvenile hormone synthesis by corpora allata of the silkworm, Bombyx mori. J. Insect Physiol. 43: 875-884.

Pratt G.E. \& Tobe S.S. 1974: Juvenile hormones radiobiosynthesized by corpora allata of adult female locusts in vitro. Life Science 14: 575-586.

RACHINSKY A. \& ToBE S.S. 1996: Role of second messengers in the regulation of juvenile hormone production in insects, with particular emphasis on calcium and phosphoinositide signaling. Archs Insect Biochem. Physiol. 33: 259-282.

Ramaswamy S.B., Shu S.Q., Park Y.I. \& Zeng F. 1997: Dynamics of juvenile hormone-mediated gonadotropism in the Lepidoptera. Arch. Insect Biochem. Physiol. 35: 539-558.
RIDDIFORD L.M. 1994: Cellular and molecular actions of juvenile hormones. I: General considerations and premetamorphic actions. Adv. Insect Physiol. 24: 213-273.

Sparagana S.P., Bhaskaran G., Dahm K. \& Ridde V. 1984: Juvenile hormone production, juvenile hormone esterase, and juvenile hormone acid methyl transferase in corpora allata of Manduca sexta. J. Exp. Zool. 230: 309-313.

Sparagana S.P., Bhaskaran G. \& Barrera P. 1985: Juvenile hormone acid methyltransferase activity in imaginal discs of Manduca sexta prepupae. Arch. Insect Biochem. Physiol. 2: 191-202.

Stay B., Tobe S.S. \& Bendena W.G. 1994: Allatostatins: Identification, primary structure, function and distribution. $A d v$. Insect Physiol. 125: 267-337.

Thompson C.S. \& Toвe S.S. 1986: Electrical properties of membranes of cells of the corpora allata of the cockroach Diploptera punctata: Evidence for the presence of voltage-sensitive calcium channels. In: Borkovec A.B. \& Gelman D.B. (eds), Insect Neurochemistry and Neurophysiology. Clifton, NJ: Humana Press, pp. 375-378.

Tobe S.S. \& PRATT G.E. 1974: The influence of substrate concentrations on the rate of insect juvenile hormone biosynthesis by corpora allata of the desert locust in vitro. Biochem. J. 144: 107-113.

Weaver R.J., Pratt G.E., Hamnett A.F. \& Jennings R.C. 1980 The influence of incubation conditions on the rates of juvenile hormone biosynthesis by corpora allata isolated from adult females of the beetle Tenebrio molitor. Insect Biochem. 10: 245-254.

Wyatt G.R. \& Davey K.D. 1996: Cellular and molecular actions of juvenile hormone. II. Roles of juvenile hormone in adult insects. $A d v$. Insect Physiol. 26: 1-156.

YAGI K.J. \& ToBE S.S. 2001: The radiochemical assay for juvenile hormone biosynthesis in insects: problems and solutions. J. Insect Physiol. 47: 1227-1234.

Received December 5, 2001; revised February 5, 2002; accepted April 23, 2002 